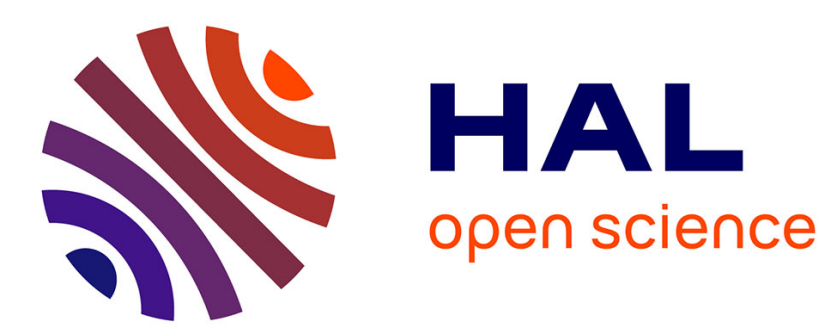

\title{
Violences envers les femmes, inégalités et situations post-coloniales
}

Dolores Pourette

\section{To cite this version:}

Dolores Pourette. Violences envers les femmes, inégalités et situations post-coloniales. Sciences Sociales et Santé, 2010, 10.1684/sss.2010.0402 . hal-01938341

\section{HAL Id: hal-01938341 \\ https://hal.science/hal-01938341}

Submitted on 28 Nov 2018

HAL is a multi-disciplinary open access archive for the deposit and dissemination of scientific research documents, whether they are published or not. The documents may come from teaching and research institutions in France or abroad, or from public or private research centers.
L'archive ouverte pluridisciplinaire HAL, est destinée au dépôt et à la diffusion de documents scientifiques de niveau recherche, publiés ou non, émanant des établissements d'enseignement et de recherche français ou étrangers, des laboratoires publics ou privés. 


\section{VIOLENCES ENVERS LES FEMMES, INÉGALITÉS ET SITUATIONS POSTCOLONIALES \\ Commentaire \\ Dolorès Pourette}

John Libbey Eurotext | « Sciences sociales et santé »

2010/4 Vol. 28 | pages 33 à 39

ISSN 0294-0337

Article disponible en ligne à l'adresse :

https://www.cairn.info/revue-sciences-sociales-et-sante-2010-4-page-33.htm

Distribution électronique Cairn.info pour John Libbey Eurotext.

(C) John Libbey Eurotext. Tous droits réservés pour tous pays.

La reproduction ou représentation de cet article, notamment par photocopie, n'est autorisée que dans les limites des conditions générales d'utilisation du site ou, le cas échéant, des conditions générales de la licence souscrite par votre établissement. Toute autre reproduction ou représentation, en tout ou partie, sous quelque forme et de quelque manière que ce soit, est interdite sauf accord préalable et écrit de l'éditeur, en dehors des cas prévus par la législation en vigueur en France. Il est précisé que son stockage dans une base de données est également interdit. 
Sciences Sociales et Santé, Vol. $28, n^{\circ} 4$, décembre 2010

\title{
Violences envers les femmes, inégalités et situations postcoloniales
}

\author{
Commentaire
}

Dolorès Pourette*

Les analyses des violences sexuelles infligées aux femmes pendant leur enfance relèvent le plus souvent d'approches psychologiques, consistant à interroger les répercussions psychologiques des violences sexuelles sur les victimes et les "profils » des agresseurs, ou d'approches démographiques mesurant la part de femmes ayant été victimes d'abus sexuels dans une population donnée et les facteurs sociodémographiques associés à ces violences. Plus rares sont les analyses socioanthropologiques qui éclairent le phénomène à la lumière des contextes socioéconomiques et politiques dans lesquels sont perpétrées ces violences, des constructions culturelles de la parenté (organisation familiale, habitat, statut de l'enfant...), de la sexualité (normes et interdits), des normes en matière de relations entre les sexes et les générations, et de leurs évolutions.

Dans cette optique, le texte de Christine Hamelin, Christine Salomon et France Lert constitue une analyse particulièrement fouillée des violences sexuelles subies par les femmes dans leur enfance au sein de la communauté kanake de Nouvelle-Calédonie. Fortes de données quantitatives issues d'une enquête statistique et de connaissances approfondies de la société néocalédonienne acquises au fil de nombreuses années de

\footnotetext{
* Dolorès Pourette, anthropologue, Centre Populations et Développement (CEPED), UMR 196 IRD-INED-Paris Descartes, France ; dolores.pourette@ceped.org
} 
recherches, les auteurs examinent le phénomène à la lumière des contours historiques, sociaux et familiaux du monde kanak.

En centrant leur analyse sur une communauté autochtone caractérisée par une histoire coloniale douloureuse et subissant jusqu'à aujourd'hui des disparités socioéconomiques et des inégalités de santé massives, les auteurs mettent en évidence comment la colonisation a contribué à une déstructuration de l'organisation économique et sociale kanake et à une marginalisation politique et socioéconomique des populations locales. La vie familiale des Kanaks s'en est trouvée particulièrement affectée : outre la pauvreté, les familles sont souvent confrontées à l'alcoolisme et à des tensions, disputes et bagarres entre conjoints. Or, ces phénomènes apparaissent comme des facteurs associés au risque d'exposition à des violences sexuelles pour les enfants, en Nouvelle-Calédonie, mais aussi dans d'autres contextes (Widmer et Pourette, 2009). Par ailleurs, le texte met en évidence les évolutions récentes du monde kanak et, notamment, la dénonciation de plus en plus fréquente des violences sexuelles subies par les femmes dans l'enfance, signe d'un abaissement du seuil de tolérance des abus sexuels sur les enfants, même quand ils sont perpétrés par un membre de la parenté.

Ces constatations m'invitent à quelques réflexions sur les violences sexuelles exercées à l'encontre des femmes pendant leur enfance dans un autre contexte postcolonial, celui de la Polynésie française (1). L'histoire de la Polynésie française (2) a été marquée par un siècle de colonisation européenne, puis, en 1962, par l'implantation du Centre d'expérimentation du Pacifique (CEP) connu pour ses essais nucléaires dans le Pacifique (3). Ces événements ont fortement bouleversé les structures sociales, économiques, politiques et religieuses de la société polynésienne. Du fait de l'arrivée de Chinois et surtout de Français, pour une durée temporaire (pour les fonctionnaires) ou plus longue, la structure de la population s'est trouvée profondément modifiée. Aujourd'hui, la population de la Polynésie française se compose de quatre groupes distincts : les Polynésiens descendants des Ma'ohi (66 \% de l'ensemble), les "Demis », issus des métissages entre Polynésiens, Européens et Chinois (17\%), les Chinois ou Tinito (5\%), les Européens ou Popaa (12\%) (Lextreyt, 2003). Comme en Nouvelle-Calédonie, les disparités socioéconomiques entre ces groupes sont très marquées et se portent en défaveur

(1) La Polynésie française a le statut de collectivité d'Outre-Mer.

(2) Archipel composé de 118 îles, dont la plus grande, Tahiti, réunit $70 \%$ de la population totale.

(3) Le retrait du CEP a eu lieu en 1996. 
des populations locales. Ainsi, en 1988, les ménages européens et assimilés représentaient $72 \%$ des cadres et professions intellectuelles supérieures et $43 \%$ des professions intermédiaires, alors que cette population ne constituait que $20 \%$ des ménages. Les ménages polynésiens (58\% du total des ménages) représentaient seulement $10 \%$ des cadres et professions intellectuelles supérieures, mais $82 \%$ des agriculteurs et $76 \%$ des travailleurs « cols bleus » (Poirine, 1992, cité par Schuft, 2007).

L'implantation du CEP au début des années 1960 a entraîné une restructuration du marché de l'emploi, qui semble avoir marqué différemment les hommes et les femmes. Alors que ces dernières ont pu trouver leur place dans des emplois du secteur tertiaire, les métiers masculins traditionnels (pêche, agriculture...) sont devenus des activités annexes. Les hommes qui les exercent encore ont ainsi vu leur statut social se dévaloriser, et nombre d'entre eux se sont trouvés d'une certaine façon dépossédés de leur fonction de chef de ménage. Pour maintenir cette place au sein de la famille et dans la société, nombreux sont ceux qui effectuent un repli identitaire sur les aspects "virils traditionnels » de la culture polynésienne (Brami Celentano, 2002 ; Saura, 1988). Se sentant moins favorisés que les femmes dans cette société, certains hommes développent des comportements de réassurance de leur position dominante, et notamment une forme de jalousie pouvant aller jusqu'à la violence conjugale. Ce décalage entre la position sociale des hommes et celle des femmes s'exprime aussi dans la moindre capacité des hommes à verbaliser leurs sentiments et leurs difficultés (Pourette, 2003). L'importance de la consommation d'alcool, qui est une forme de lien social pour les hommes et pour les femmes, tend à accentuer ces phénomènes (Grépin, 2001). De plus, la quasi-omniprésence du groupe familial qui contrôle les faits et gestes de chacun(e) dans le sens du maintien des traditions est renforcée par l'insularité, l'exiguïté de l'espace où « tout se sait » (Jaspard et al., 2004).

Les enquêtes sur les violences envers les femmes en Polynésie française (4), réalisées en 2002 dans la continuité de l'enquête ENVEFF (Enquête sur les violences envers les femmes en France) (Jaspard et al., 2003a), mettent en évidence des taux de violences proches de ceux observés en Nouvelle-Calédonie (et bien plus élevés que les taux relatifs à la France hexagonale) (5). Pour ce qui est des violences sexuelles subies par

(4) Une enquête quantitative réalisée en face à face auprès de 1001 femmes, et une enquête qualitative menée auprès de 35 femmes ayant été victimes de violences et de 10 hommes auteurs de violences.

(5) En métropole, le taux d'agressions sexuelles avant 15 ans est de $4 \%$. 
les femmes pendant leur enfance, $7 \%$ des femmes ont subi au moins une agression sexuelle avant leur quinzième anniversaire. Certaines ont cumulé deux, voire trois, types d'agressions ; ainsi, $5 \%$ ont été forcées à faire ou à subir des attouchements, dont près d'un tiers plus de trois fois, $2,5 \%$ ont été victimes d'une tentative de viol (un tiers plusieurs fois) et $1 \%$ ont été violées (une sur deux au moins deux fois). Les auteurs de ces agressions sont majoritairement des hommes de la famille ou de l'entourage proche, ce qui rend très difficile les dénonciations. Ces proportions de femmes ayant subi des sévices sexuels dans leur jeunesse ne varient pas significativement selon les générations (contrairement à ce qu'on observe en Nouvelle-Calédonie). Cependant, elles sont beaucoup plus élevées chez celles qui ont rencontré d'autres difficultés durant l'enfance : $4 \%$ des femmes exemptes de brutalités physiques dans l'enfance et l'adolescence ont subi des agressions sexuelles avant leurs 15 ans, contre $11 \%$ de celles qui ont été brutalisées quelquefois et $17 \%$ de celles qui l'ont été souvent ou très souvent (Jaspard et al., 2004).

En Nouvelle-Calédonie, les abus sexuels précoces (dont la moitié est le fait de parents) constituent un facteur d'exposition majeur aux violences sexuelles à l'âge adulte, et ce dans tous les cadres de vie. Parmi les femmes qui ont subi des violences sexuelles dans l'enfance, $20 \%$ déclarent au moins une agression sexuelle au cours de l'année précédent l'enquête (contre $8 \%$ des autres) (Hamelin et Salomon, 2007). La répétition des violences est également attestée en Polynésie française, puisque 3 femmes sur 10 victimes d'au moins une agression sexuelle avant 15 ans l'ont aussi été après (contre $6 \%$ de celles qui ont été épargnées avant 15 ans) (Jaspard et al., 2003b). Ces phénomènes de répétition sont largement mis en évidence et conduisent à soutenir l'hypothèse de la reproduction de la violence, hypothèse selon laquelle les victimes et les auteurs reproduisent les situations de violence qu'ils ont vécues dans leur enfance (Jaspard et al., 2003c). Cependant, on peut se demander si le fait d'avoir subi dans son enfance certaines formes d'atteintes ne favorise pas la verbalisation de celles-ci lorsqu'elles se perpétuent à l'âge adulte. Ainsi, l'une des femmes enquêtées en Polynésie a pu parler des viols à répétition qu'elle a subis dans son enfance par son père lorsqu'elle s'est à nouveau fait agresser, à l'âge adulte. Cette nouvelle agression sexuelle lui a permis d'exprimer ce qu'elle avait tu jusqu'alors. Ce cas ne semble pas isolé puisque, selon une conseillère du service juridique d'aide aux victimes, environ 9 femmes polynésiennes sur 10 qui portent plainte pour agression sexuelle à l'âge adulte auraient déjà été victimes de viols incestueux dans leur enfance. " En règle générale ce sont des jeunes femmes, qui ont entre 20 et 30-35 ans, et qui (...) ont été victimes de viols dans leur enfance, par le père, par un cousin, enfin par quelqu'un de la famille. Là c'est prati- 
quement à $90 \%$. Je parle des femmes polynésiennes. Des choses qui n'ont pas été parlées, des incestes qui n'ont pas été parlés, des viols intrafamiliaux qui n'ont pas été parlés, et systématiquement il y a un viol, à l'âge adulte, pas forcément par quelqu'un de proche, par quelqu'un d'extérieur. Et à ce moment là, elles peuvent en parler » (Pourette, $2002: 24$ ).

Même si les condamnations pour violences faites aux femmes et pour délits sexuels ont connu une progression exponentielle depuis le début des années 2000 en Polynésie (près des deux tiers des hommes condamnés par la justice le sont pour des crimes sexuels), les témoignages de femmes ayant subi des abus sexuels dans leur enfance montrent les réticences familiales qu'elles ont rencontrées pour que ces actes soient pris en compte et sanctionnés. Les incestes subis n'étaient pas forcément " cachés » au sens où certaines femmes en avaient parlé à un membre de leur famille (leur mère, par exemple) ou savaient que les agressions commises étaient connues des proches, présents au moment des faits (6). Cependant, le secret n'a jamais été dévoilé au-delà du groupe familial restreint, probablement en raison de la crainte du jugement social, mais également en raison des répercussions d'une déclaration à la justice, d'un jugement, puis d'un emprisonnement. Les conditions de vie de nombreuses familles, qui ne peuvent parfois compter que sur le revenu de l'homme, peuvent expliquer cette réticence à dénoncer ces abus sexuels (7). Son incarcération signifie en effet la privation d'un revenu, dans un contexte où les aides sociales sont restreintes et où l'allocation de chômage n'existe pas.

Si l'enquête quantitative sur les violences envers les femmes en Polynésie française ne permet pas de distinguer l'appartenance ethnique des femmes (8), les Polynésiens (et dans une moindre mesure les Demis),

(6) L'enquête a montré que le fait de vivre dans un groupe familial élargi, sur les terres ou sous le même toit que la famille ou la belle-famille est loin de protéger les femmes des violences conjugales : en cas de cohabitation, toutes les formes de violences conjugales (psychologiques, physiques, sexuelles) deviennent plus fréquentes (Jaspard et al., 2004). Mon hypothèse est que la cohabitation entre des enfants et des membres masculins de la parenté (oncle, beau-père, cousin...) favorise le passage à l'acte et les abus sexuels sur les enfants, dans des contextes de précarité marquée et d'alcoolisation fréquente.

(7) Dans une étude sur les prisonniers incarcérés pour inceste à Tahiti, Pottier (1997) signale que les familles se plaignent de la longueur de la peine et prévoient un retour des hommes incestueux dans leur famille.

(8) Quatre-vingt-cinq pour cent des femmes interrogées se déclarent polynésiennes. Les femmes interviewées au cours de l'enquête qualitative sont toutes polynésiennes. 
qui constituent les groupes les plus marginalisés économiquement, sont particulièrement touchés par ces conditions de vie et d'habitat exposant les enfants et les femmes à des risques de violences sexuelles et rendant d'autant plus difficiles les démarches de dénonciation. Ces remarques soulignent la nécessité de poursuivre les études postcoloniales prenant notamment en compte les effets de la colonisation sur les relations familiales et sur les rapports de genre au sein des populations locales. Elles invitent également à une réflexion sur la légitimité de tenir compte de l'appartenance ethnique des populations enquêtées pour une analyse fine des répercussions des processus historiques et coloniaux sur les conditions de vie et les relations sociales contemporaines.

\section{RÉFÉRENCES BIBLIOGRAPHIQUES}

Brami Celentano A., 2002, La jeunesse à Tahiti : renouveau identitaire et réveil culturel, Ethnologie Française, 4, 647-661.

Grépin L.H., 2001, L'adolescence masculine aux Tuamotus de l'est aujourd'hui. Le taure'are'a : contradictions et transformations d'une catégorie sociale traditionnelle, Thèse de doctorat en anthropologie sociale, EHESS, Paris.

Hamelin C., Salomon C., 2007, Violences et familles en Nouvelle-Calédonie. Perspectives ethnographiques et statistiques, In : Jaspard M., Chetcuti N., $e d s$, Violences envers les femmes. Trois pas en avant, deux pas en arrière, Paris, L'Harmattan, 257-272.

Jaspard M., Brown E., Condon S., Fougeyrollas-Schwebel D., Houel A., Lhomond B., Maillochon F., Saurel-Cubizolles M.J., Schiltz M.A., 2003a, Les violences envers les femmes en France. Une enquête nationale, Paris, La Documentation Française.

Jaspard M., Brown E., Pirus C., 2003b, Enquête quantitative sur les violences envers les femmes en Polynésie française, Paris, Institut de Démographie de l'Université Paris 1.

Jaspard M., Brown E., Lhomond B., Saurel-Cubizolles M.J., 2003c, Reproduction ou résilience : les situations vécues dans l'enfance ont-elles une incidence sur les violences subies par les femmes à l'âge adulte?, Revue Française des Affaires Sociales, 3, 159-190.

Jaspard M., Brown E., Pourette D., 2004, Les violences envers les femmes dans le cadre du couple en Polynésie française, Espaces, Populations et Sociétés, 2, 325-341. 
Lextreyt M, 2003, Polynésie française : un modèle socioculturel spécifique, In : de Deckker P., ed., L'outre-mer français dans le Pacifique. NouvelleCalédonie. Polynésie française. Wallis-et-Futuna, Paris, L'Harmattan, 129134.

Poirine B., 1992, Tahiti : Du melting-pot à l'explosion ?, Paris, L'Harmattan. Pottier P., 1997, Choc des cultures et justice pénale. Loi, prison et inceste à Tahiti, Mémoire de DEA, Université Française du Pacifique, Tahiti.

Pourette D., 2002, Les violences envers les femmes en Polynésie française. Étude socioanthropologique, Paris, Laboratoire d'Anthropologie Sociale ; Papeete, Direction de la Santé.

Pourette D., 2003, Des mots aux maux. Les violences conjugales en Polynésie française, Gradhiva, 33, 77-83.

Saura B., 1988, Culture et renouveau culturel, In : Gleizal C., ed., Encyclopédie de la Polynésie. Vivre en Polynésie, T.9, Papeete, 57-72.

Schuft L., 2007, Attitudes et intégration sociale des fonctionnaires métropolitains à Tahiti, Bulletin de la Société des Études Océaniennes, 309, 75-104.

Widmer I., Pourette D., 2009, Les violences envers les femmes à l'île de la Réunion. Poids des chiffres, paroles de victimes, Aix en Provence, Publications de l'Université de Provence.

Conflit d'intérêts : aucun. 